\title{
The effects of stimulus context on the time required for choosing the larger or the smaller of two digits
}

\author{
CARL BENNINK \\ Grand Valley State Colleges, Allendale, Michigan 49401
}

\begin{abstract}
Evidence currently exists which suggests that adaptation-level (AL) theory (Helson, 1964) is a concept relevant to the judging of numerical inequalities. The present experiment was designed to replicate the findings of Fairbank and Capehart (1969) that subjects require less time to choose the larger of two digits than to choose the smaller. In addition, the prediction was made on the basis of AL theory that manipulating stimulus context would influence the time required for subjects to select the larger or the smaller of two digits. In one condition of a simultaneous comparisons task, the digit 2 was interpolated midway between each stimulus pair. In another condition, the digit 9 was the interpolated digit. Subjects required less time to choose the larger of a pair of digits when 2 was the interpolated digit and less time to choose the smaller of a pair when 9 was the interpolated digit. The results were discussed in terms of $\mathrm{AL}$ theory and suggestions for further research were proposed.
\end{abstract}

Moyer and Landauer (1967) 'found that the time required to discriminate the larger of two digits depends on their numerical difference. Specifically, it was found that the larger the difference, the shorter was the time required. The results were interpreted as suggesting that the psychological process involved in the judgment of numerical values is analogous to that involved in the judgment of physical magnitudes.

These results led Fairbank and Capehart (1969) to test the applicability of adaptation-level (AL) theory (Helson, 1964) to the judging of numerical inequalities. Consistent with their predictions, it was found that the time required for selecting the larger of a pair of digits is shorter than the time required for selecting the smaller of the pair. That this finding could be successfully predicted on the basis of AL theory derives from the following considerations.

According to $\mathrm{AL}$ theory, stimuli are judged with respect to the prevailing adaptation level of the organism to a given set of stimuli. In addition, the psychological contrast of any stimulus is a function of the distance between that stimulus and the prevailing AL. The prevailing $\mathrm{AL}$, equated with the stimulus value eliciting a neutral response, is typically displaced below the arithmetic mean of a set of stimuli. Thus, the larger member of a stimulus pair would have greater contrast with respect to $\mathrm{AL}$ than the smaller. When decision time is taken as a measure of psychological contrast (Capehart \& Pease, 1968), it follows that subjects should require less time for selecting the larger of a pair of stimulus items than for selecting the smaller.

Typically, an approximation to $\mathrm{AL}$ is defined as the

The author wishes to thank Alfred B. Kristofferson for sponsoring this paper and taking editorial responsibility for it and Thomas R. Herzog for his assistance in preparing the manuscript.

Requests for reprints should be sent to Carl Bennink, who is now at the Department of Psychology, University of Louisville, Louisville, Kentucky 40208. weighted logarithmic mean of the series, context, and residual stimuli. Series stimuli are taken to be those stimuli introduced into a stimulus field for comparison and may be referred to as "attended stimuli." Series stimuli, then, are the focus of response. Context stimuli form the background against which the attended stimuli are perceived, and their influence on AL usually derives from such determinants as intensity, novelty, frequency, etc. Past experience with similar stimuli is classed as residual stimulation. Thus, AL represents the pooled effect of each class of stimuli and shifts in AL occur with changes in any of the three classes of stimuli in proportion to their weighted contributions in a particular situation. It follows that the introduction of stimuli above or below the prevailing AL of the organism results in a corresponding shift in AL in the direction of the new stimulus value provided the stimulus is of sufficient intensity relative to the existing $\mathrm{AL}$.

The present experiment was designed to replicate that of Fairbank and Capehart (1969) and also to test the possibility that manipulating context by introduction of an interpolated digit in the attended pair would affect the decision time for comparative judgments. More specifically, the introduction of an interpolated digit close, on the average, in numerical value to the prevailing $\mathrm{AL}$ of a stimulus pair should result in shorter decision time for choosing the larger of the attended pair of digits. On the other hand, introducing a digit far enough above the $\mathrm{AL}$ in value should render exactly the opposite result, i.e., the prevailing $\mathrm{AL}$ should be shifted upwards and the smaller digit of the attended pair should then have greater contrast with reference to the prevailing AL. For this task, the digit 2 was taken to be close in numerical value to the prevailing AL of the pairs of single digits and the digit 9 was assumed to be sufficiently above the AL in value to result in an upward displacement. 


\section{METHOD}

\section{Subjects}

Thirty-six students (23 females and 13 males) from the introductory psychology class of The Grand Valley State Colleges volunteered to serve as subjects in partial fulfillment of course requirements.

\section{Stimulus Materials}

The complete list of 72 nonidentical pairs of digits 1-9 was randomized eight times. Two random orders were typed, in elite size, on each of four pages of white bond paper, yielding 144 pairs of digits per page. The two digits of each pair were separated by three horizontal spaces, and each pair was separated by eight spaces horizontally and double-spacing vertically. The digits were arranged on each page in six columns of 24 pairs.

Eight additional pages, using the same lists of digits, were typed in the same manner with the following exceptions: On four of the lists, the digit 2 was interpolated midway between each pair, and on the remaining four pages, the interpolated digit was 9 .

Twelve copies of each page were produced on a Xerox 1000 copier and were used to form 36 booklets of four pages each. Twelve booklets contained the digit pairs with no interpolated digit, 12 contained the interpolated digit 2 and 12 the digit 9 . Booklets were formed using the four balanced orders of pages. Each order was repeated three times in each group of 12 .

\section{Procedure}

An equal number of subjects was assigned to each of three groups. The first group (Group I) received booklets containing no interpolated digit. The second group (Group II) received booklets containing the interpolated digit 2 and the remaining group (Group III) received booklets containing 9 as the interpolated digit.

Subjects in Group I were instructed to cross out the larger or smaller, depending on instructions, of each pair as quickly as possible with a minimum of errors. Subjects were timed separately for the completion of each page by the experimenter using a stopwatch. A 1-min rest period was given between pages and each page was begun without prior exposure. Groups II and III completed exactly the same task except they chose between the first and last digits of each triplet. Thus, each subject made the choices between the same pairs of digits. Included in the instructions to Groups II and III was the following statement: "Pay attention to the middle digit as you go along because I will ask you something about it later. However, make your choice of the (larger/smaller) digit only between the first and the last."

For each group, half the subjects crossed out the larger digits on the first page, the smaller digits on the next two pages, and the larger ones on the fourth. The other half crossed out the smaller digits on the first page followed by two pages of larger digits and, finally, one page of crossing out the smaller digits.

\section{RESULTS}

The results were analyzed separately for the group choosing between a pair of digits (Group I) and the groups choosing between the first and last digits of each triple (Groups II and III). For Group I, the mean time per page for crossing out the smaller of a pair of digits was $41.9 \mathrm{sec}$, and the mean time per page for crossing out the larger of the pair was $35.2 \mathrm{sec}$. A Treatments by Subjects analysis of variance for Group I shows the difference between the time required for crossing out the larger and the smaller of a pair of digits to be
Table 1

Means of Task by Interpolated Digit Interaction

\begin{tabular}{lcc} 
& \multicolumn{2}{c}{ Interpolated Digit } \\
Task & 2 & 9 \\
\hline Small & 62.2 & 60.8 \\
Large & 53.9 & 68.8 \\
(S - L) & 8.3 & -8.0 \\
\hline
\end{tabular}

significant $[F(1,11)=22.2, p<.001]$.

The analysis of variance for Groups II and III reveals that the only significant effect is the interaction between task and interpolated digit $[\mathrm{F}(1,22)=11.8, \mathrm{p}<.005]$

Table 1 shows the means of Task by Interpolated Digit interaction. Tukey's B-test was performed using Cicchetti's (1972) adjusted q statistic based on the number of unconfounded comparisons. The critical value, based on $\mathrm{df}=22$ and $\mathrm{s}_{\overline{\mathrm{x}}}=2.298$, is 7.456 . Thus, the difference between the mean time required for crossing out the larger and the smaller digits is significant, $\mathrm{p}<.05$, for both conditions of interpolated digits.

The average error rate was less than $1 \%$ and the number of errors committed did not differ significantly for either task or interpolated digit conditions.

\section{DISCUSSION}

The present study has replicated the findings of Fairbank and Capehart (1969). In addition, the predictions made with respect to AL theory (Helson, 1964), that the manipulation of stimulus context affects the decision time for comparative judgments of numerical stimuli has been supported.

The results of the condition where no interpolated digit was present clearly support the assumption of a decentered $\mathrm{AL}$ for this task as Fairbank and Capehart (1969) pointed out. Central to the concept of decentering is the assumption that $\mathrm{AL}$ represents the pooled effect of all stimuli presented for judgment in addition to any residual effects. The effect of introducing an interpolated digit into the pairs of digits which were compared further illustrates that this averaging or pooling mechanism is a viable assumption in the case of numerical judgments. Hence, further support is given to the contention that AL theory (Helson, 1964) is a concept relevant to numerical stimuli.

What remains to be established are the parameters of the pooling mechanism in the case of comparative numerical judgments. This may include establishing the frequency with which the interpolated digit must appear in order to maintain a given level of adaptation. In addition, the effect of moving the interpolated digit outside of the pairs to be judged might be investigated.

More detailed predictions could be made if the exact shape of the function relating judgment time to interpolated digit could be established for a broad range of numerical stimuli. For example, different results might be obtained when multiple-digit and fractional numbers are judged as opposed to the single-digit whole numbers used in the present study.

It is suggested that future studies be carried out using a controlled viewing apparatus such as a tachistoscope. This would allow for separate measures of judgment time for each pair or triple of the stimulus set. With separate measures, one could, for example, determine the degree to which $A L$ is shifted on each successive presentation. In addition, controlled viewing would allow for the separate presentation of focal and background 
stimuli for varying amounts of time, and thus yield more precise measures of the relative contributions of each of these classes of stimuli to the prevailing AL of the numerical stimulus set.

\section{REFERENCES}

Capehart, J., \& Pease, V. An application of adaptation-level theory to transposition responses in a conditional discrimination. Psychonomic Science, 1968, 10, 147-148.

Cicchetti, D. V. Extension of multiple-range tests to interaction tables in the analysis of variance: A rapid approximate solution. Psychological Bulletin, 1972, 77, 405-408.

Fairbank, B. A., Jr., \& Capehart, J. Decision speed for the choosing of the larger or the smaller of two digits. Psychonomic Science, 1969, 14, 148.

Helson, $\mathrm{H}$. Adaptation level theory, An experimental and systematic approach to behavior. New York: Harper \& Row, 1964.

Moyer, R. S., \& Landauer, T. K. Time required for judgment of numerical inequality. Nature, 1967, 215, 1519-1520.

(Received for publication October 7, 1974.)

Bulletin of the Psychonomic Society

1975, Vol. 5 (1), 20-22

\title{
Forward and backward associations among serial list items*
}

\author{
J. D. RE AD \\ University of Lethbridge, Lethbridge, Alberta, Canada T1K $3 M 4$
}

\begin{abstract}
Following serial learning, a paired-associates learning task in which the pairs were comprised of adjacent serial list items assessed the relative strengths of forward and backward associations between pair members. In addition, either one or two items served a stimulus function within the pairings. The results suggested that forward associations were relatively stronger at the beginning of the serial list than backward associations and that the reverse of this relationship was true over the latter half of the serial list. Moreover, the results suggested that compound associations were relatively stronger over the last half of the serial list than two-item associations, and the reverse of this relationship was true at the beginning of the serial list. The results were related to Lashley's (1951) interpretation of sequential behavior.
\end{abstract}

Throughout the history of research concerned with the associations formed between members of a serial list, backward associations have generally been neglected in favor of an interest in forward associations. For example, in virtually all of the serial learning (SL) to paired-associates learning (PAL) and PAL to SL transfer studies designed to test hypotheses about the functional stimuli in SL, construction of the transfer lists was based upon presumed forward associations between serial item or positions. However, in those studies using

*This research was supported by Grant A8350 from the NThis research was supported by Grant A8350 from the Lethbridge Research Grant 28-1069. The author wishes to thank Stuart Travis and Richard Peterson for their assistance in data collection. Requests for reprints should be sent to J. D. Read, Department of Psychology, University of Lethbridge, Lethbridge, Alberta, T1 K 3M4. double-function PA lists, the generally slower learning and negative transfer effects observed of ten have been attributed to the theoretical presence of backward associatons which arise as a result of PA items serving both stimulus and response functions (e.g., Postman \& Stark, 1967). As yet there have been no investigations of the relative strengths of forward and backward associations at the conclusion of an SL task.

In his classical paper on the problem of serial order in behavior, Lashley (1951) expressed his belief that the individual components of a verbal or motor activity are determined more by the idea to be expressed or the intention to act than by the specific chain of successive stimuli and responses. In other words, if that which has yet to be expressed determines in some way that which precedes it, an extension of Lashley's hypothesis to a verbal SL task might suggest that those items in 